\title{
Experimental uncertainty budget for concrete compressive strength test based on a multifactorial analysis
}

\author{
Stamatia Gavela, Nikolaos Nikoloutsopoulos, George Papadakos, Dimitra Passa, \\ Anastasia Sotiropoulou \\ School of Pedagogical and Technological Education, Department of Civil Engineering Educators \\ matina@gavela.gr, http://orcid.org/0000-0003-0775-2750; nikolasnikoloutso@hotmail.com; gpapadakos@tteemail.gr; \\ dimpassa@aspete.gr; sotiropoulou@aspete.gr
}

\begin{abstract}
The objective of the study is to introduce an experimental uncertainty budget process for concrete compressive strength test, based on a protocol that incorporates effects of multiple factors significant for the measurement result. The proposed procedure is rather useful for laboratories seeking accreditation according to ISO/IEC 17025, in order to emphasize the contribution of type A uncertainty estimations, rather than relying on type B estimations that are unable to address the correlation between those factors. Two independent experiments were performed. Experiment I is proposed as a simple, suitably designed, reproducibility trial for laboratories performing EN 12390 test method, i.e. when a specified nominal curing age is targeted, following experimental design on multiple uncertainty parameters. A sensitivity analysis was introduced based on a semi-empirical multifactorial regression model (experiment II) for concrete compressive strength as a function of specimen's curing age and $\mathrm{W} / \mathrm{C}$ ratio. The present study is an effort towards an integrated and standardized method for experimental, semi-empirical multifactorial regression estimation of the uncertainty budget for the EN 12390 test method, being useful, also, as a baseline for internal quality control programs when adjusted for the specific characteristics of concrete specimens tested by a laboratory.
\end{abstract}

KEYWORDS. Concrete compressive strength test; Multifactorial model; Type A uncertainty estimation; Sensitivity analysis.

\section{open 1 access}

Citation: Gavela, S., Nikoloutsopoulos, N., Papadakos, G., Passa, D., Sotiropoulou, A. Experimental uncertainty budget for concrete compressive strength test based on a multifactorial analysis, Frattura ed Integrità Strutturale, 50 (2019) 383-394.

Received: 19.01.2019

Accepted: 14.05.2019

Published: 01.10.2019

Copyright: (C) 2019 This is an open access article under the terms of the CC-BY 4.0, which permits unrestricted use, distribution, and reproduction in any medium, provided the original author and source are credited.

\section{INTRODUCTION}

$\mathrm{D}$ etermination of the uncertainty budget in cases of measuring the compressive strength of concrete specimens is critical as it is a quantitative criterion according to which the result of a corresponding test is assessed against the compliance of the specimen with concrete specifications. This is particularly emphasized in the new version of International Standard ISO/IEC 17025:2017, by which it is required that any conformity assessment laboratory output 
shall be provided according to a predetermined decision rule. Most of the attempts to determine the uncertainty budget for a concrete compressive strength test according to European Standard EN 12390-3 are based mainly on Type B estimations, i.e., on external laboratory sources or on reasonable assumptions according to scientific knowledge. Type B estimation of uncertainty factors is simple and fast and provides a solution in cases where it is not feasible to perform an experimental (statistical) uncertainty investigation (a type A estimation). This type of assessment leads to a number of disadvantages, such as the inability to assess the degree of positive or negative correlation between the various parameters that contribute to the result of the measurement.

In this study, firstly the parameters of uncertainty during an EN 12390-3:2009 testing for specimens prepared according to EN 12390-1: 2012 are analyzed using an Ishikawa (cause and effect) diagram. Two experiments were performed for further statistical investigation of the interacting behavior of the most essential parameters presented in the Ishikawa diagram. The first experiment is also proposed as a procedure for a laboratory performing the EN 12390 test method in order to reveal its type A estimation for the most essential corresponding uncertainty parameters when a specified nominal curing age is targeted. Within this framework it is essential to perform a sensitivity analysis on the effect of the specimen's curing age. To do so, a semi-empirical model, was estimated within the second experiment for the correlation of concrete compressive strength as a function of the parameters of specimen's curing age and W/C ratio. The proposed procedure is described in a way to be exploitable by any laboratory, especially in the case of seeking accreditation according to ISO/IEC 17025. The semi-empirical model is expected to be useful, also, for accredited testing laboratories in order to perform their internal quality control program.

\section{UNCERTAINTY PARAMETERS IDENTIFICATION AND ANALYSIS}

$\mathrm{T}$ he characteristics of the specimen that is subjected to a compressive strength measurement according to European Standard EN 12390-3 are subject to a definition detailed in EN 12390-1. Any deviation from the technical specifications of the specimen, i.e. in terms of its geometrical and other characteristics, also leads to a measurement error. This does not affect directly the numerical quantity resulting from the application of the compressive strength test method if a proper testing apparatus is used. It occurs indirectly, as any measurement result is attributed to a theoretically perfect cube with edges that are all of equal length, e.g. exactly $15 \mathrm{~cm}$. This is something that cannot be achieved perfectly, in practice. By testing as many specimens of the same characteristics as possible, is expected to yield measurement results dispersion that is affected by these definitional deviations. Such errors contribute to the overall uncertainty of the test result inherently. They are impossible to be eliminated, but it is possible to be minimized [1] by improving the preparation conditions of the specimens (e.g. by improving the manufacturing quality of the moulds being used by the laboratory).

The category of factors contributing, due to definitional errors, to the increase of the combined measurement uncertainty include, also, those associated with the definition of the aimed concrete composition for the specimen prepared, and of course, of the concrete used in the corresponding construction. The values for the mix proportions of the concrete constituents, usually expressed in $\mathrm{kg} / \mathrm{m}^{3}$, and the particle size of the aggregates used are two of these factors. In particular, however, the ratio of the amount of water to the amount of cement used in the concrete mix has already emerged from the late 19th century as essential for concrete compression strength test results. Mathematical models that value this relationship for a given specimen curing age have been early proposed [2,3]. Recent work has suggested mathematical models describing the apparent association of the specimen compressive strength with curing age [4-11].

Another factor that affects the result of concrete compressive strength for a specimen of a given curing age is the temperature of the environment in which the curing process takes place [5]. It is characteristic that a higher value of this temperature leads to faster curing. At the nominal age (e.g. on 28 days) the specimen may exhibit different value of compressive strength as compared to the case where the same curing procedure would be performed in a lower temperature environment.

Of course, the combined uncertainty of the result of a test according to EN 12390-3 depends on the calibration quality of the uniaxial compression apparatus and the degree of familiarity of its user. An apparatus that has been successfully calibrated according to the requirements of International Standard ISO 7500-1 may be considered as causing negligible random errors to the test results. However, it should be noted that any systematic errors due to the accuracy of the reference standard (e.g. a force gauge) which is used during the calibration procedure cannot be overlooked. For this reason, the measurement uncertainty for the reference standard should always be incorporated into the uncertainty budget of a test according to EN 12390-3.

All of the above are summarized in the cause-and-effect diagram (Ishikawa diagram, as already been introduced by authors of this paper in a previous study [8]) which has been used according to guidance from EURACHEM [11], in a way [8,12] that aims at mapping uncertainty factors while simultaneously visualizing synergies between them (Fig.1). 


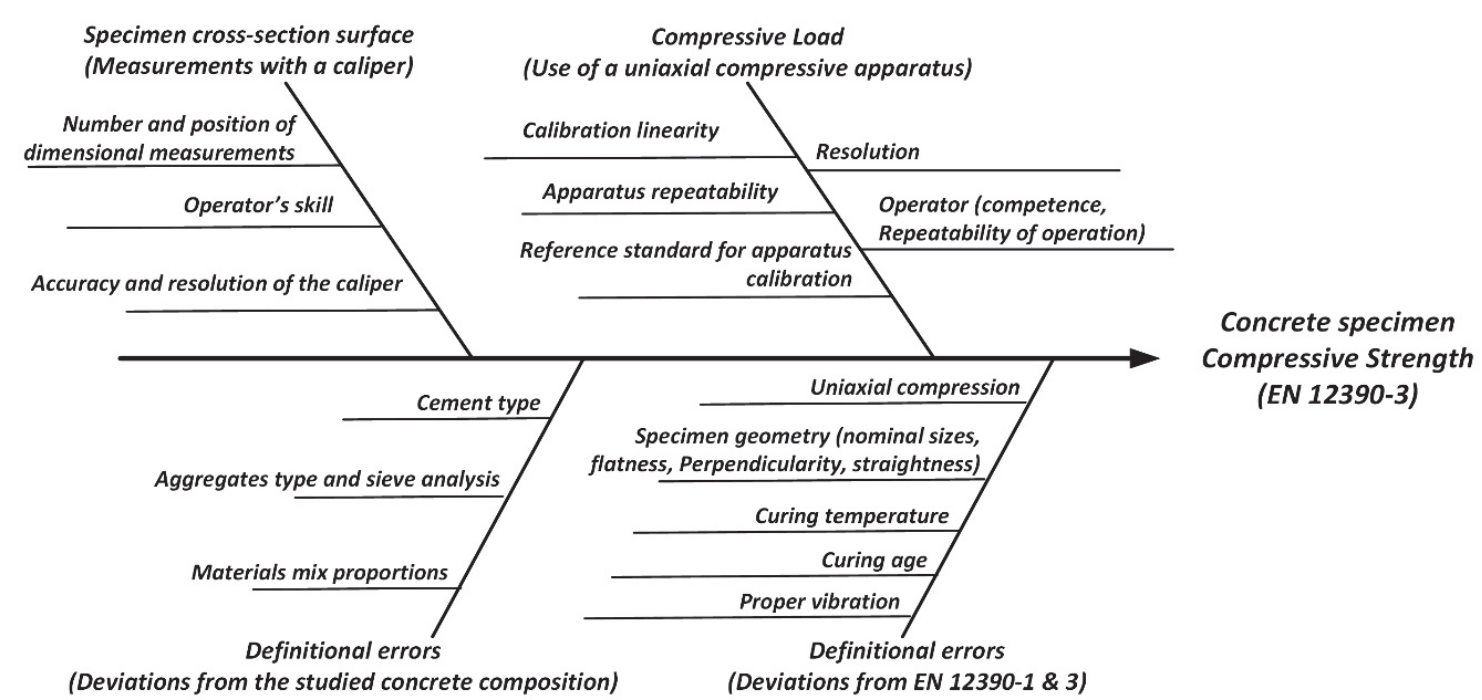

Figure 1: Cause-and-effect diagram on how uncertainty factors contribute to the final result of a test according to EN 12390-3.

\section{SCOPE OF THE EXPERIMENTS}

\section{Scope of experiment I}

7 actors like equipment accuracy, testing method selection, e.g. the EN 12390-3 method, measurement conditions -1 and equipment handler's skills should be considered as inherent to the operation characteristics of the laboratory. 1 Their reproducibility is a matter under control of the interested laboratory. A simple reproducibility experiment, in which the same equipment is used for all the needed measurements, provides adequately the contribution of all these parameters to the laboratory's uncertainty estimation, as the specific laboratory will continually use the specific equipment, under the specific measurement conditions, by the same handlers. However, a simple reproducibility experiment cannot incorporate the effect of parameters, that have to do with the specimen's characteristics (which define the concrete composition under consideration), especially when the specimen is not prepared by the laboratory. Such parameters are all those related to the mix composition and to the definitional uncertainty produced by deviations from the definition given for the specimen in EN 12390-1, especially its geometrical characteristics.

In experiment I of this study, the experimental design incorporated different levels of cement content (CC) and water to cement ratio $(\mathrm{W} / \mathrm{C})$, which is adequate in order to produce the majority of the corresponding combinations of mix constituents that the laboratory could encounter when receiving specimens of a specific combination of types of concrete constituents. By fixing the type of the materials for the proposed type A uncertainty estimation makes this protocol materials' type specific. This is not considered to reduce its effectiveness, especially when a laboratory receives specimens from a specific producer on an ongoing basis.

In real circumstances of laboratory operation, small deviations of maximum three days from the nominal curing age of performing the compressive strength test may occur for any reason. A stratification of such deviations was also included in the design of the experiment in order to produce such an uncertainty factor. Also, a large number (about 30) of different molds, of three different construction designs, where used randomly for the preparation of the specimens, providing varying geometries, representative to the corresponding variation that a laboratory could encounter when receiving any specimen from any external customer.

The aim is to propose a method for determining the uncertainty for an EN 12390-3 concrete compressive strength test result, the experimental part being extended, but only in the case where the test method is applied for a specific nominal value of curing age. The method is formulated in such a way that it is reproducible and exploited by any laboratory that applies this test, especially if it seeks accreditation in accordance with ISO / IEC 17025. This protocol of the proposed method for a type A uncertainty estimation is designed in such a way that it leads to:

(a) the optimum economy of used specimens,

(b) the aggregation of covariation among all the parameters that vary due to the experimental design, and

(c) a (safe-side) maximal estimation of the uncertainty budget, something that is, also, supported by ISO in the GUM approach. 
Scope of experiment II

Experiment II aims at the experimental investigation of the correlation of compressive strength testing results with the parameters of curing age and water to cement ratio at the same time, through sensitivity analysis. A sigmoidal curve was used to fit the experimental results. Freiesleben Hansen \& Pedersen [4] and Carino [5] firstly proposed a sigmoidal curve for modeling the curing procedure.

All of the parameters presented in Fig.1 affect the compressive strength testing according to European Standard EN 12390 series. Some essential questions are: to what extent do all these parameters correlate to each other and to the result of the test procedure? Are the results of the testing procedure valid if these parameters fail to be accurately determined? For example, should a testing result be put aside if the curing age of the specimen deviates by a few days from the typical nominal 28-days value?

The population of parameters affecting the result of a compressive strength test is big so unless performing sensitivity analysis for any subset of these parameters via a specialized experiment it is almost impossible to assess the impact of this subset of parameters. Studying the effect of all the above parameters in one single experiment for various levels of those parameters would lead to an enormous specimens' population. For this reason in the frame of an extended study aiming at the creation of a function that correlates the testing result on the compressive strength of concrete specimens to all the significant of the above parameters, only the experimental investigation of the correlation of compressive strength testing results with the parameters of curing age and water to cement ratio was examined. The integration of various similar experiments of such a protocol by various laboratories and for various parameters of the test procedure could speed the achievement of a standardized semi-empirical model on the relation of concrete compressive strength as a function of a great number of testing parameters and mix materials characteristics [8].

\section{EXPERIMENTAL DESIGN}

I $\mathrm{n}$ the context of this study, tests have been carried out on a number of specimens prepared according to the definition of a $15 \mathrm{~cm}$ cementitious concrete test specimen as set out in EN 12390-1. For the design of the experiments a combination of characteristics for the composition of the concrete was chosen for the preparation of the specimen. The cement used in the present study was CEM II 32.5. The aggregates used were crushed limestone. Determination of particle density and water absorption of fine and coarse aggregates was performed according to EN 1097-6. The superplasticizer used was Sika ViscoFlow 700. The concrete compositions of the tested specimens are shown in Table 1 as $\mathrm{kg}$ of constituent per $1 \mathrm{~m}^{3}$ of fresh concrete produced $\left(\mathrm{kg} / \mathrm{m}^{3}\right)$. Those compositions were obtained after performing concrete mix design using the densities of raw materials. Concrete mix design can lead to errors when raw materials are "fluffy" or lightweight or can absorb big amounts of water [10] because for these materials the error in the determination of its density is bigger but also because of a degree of compaction as the air within the initial amount of this "fluffy" material (before mixing) is displaced by all the other constituents of the mixture (during mixing). In this study, conventional aggregates were used so the errors are expected to be small. Deviations in mix proportions have been addressed to the factors contributing to the uncertainty in Ishikawa diagram above. If a laboratory reproduces the experiments using concrete mix design (using densities of raw materials) will address the same systematic errors. However, the fresh concrete's density can be determined according to EN 12350-6. Knowing the density of fresh concrete, the yield per batch can be determined as the mass of all the ingredients in a batch divided by the density. In this way one can verify if proportions of ingredients that came from concrete mix design $\left(\mathrm{kg} / \mathrm{m}^{3}\right)$ produces indeed $1 \mathrm{~m}^{3}$ of fresh concrete.

In experiment I, the uncertainty estimation process was performed for the case of applying the 28-day nominal curing age that is set by the corresponding national regulation on structural concrete technical specifications. For each of the experiment I compositions, ten specimens were prepared and tested for compressive strength by pairs, at curing ages equal to 27,28 , 29, 30 and 31 days, respectively. Experiment II was intentionally performed using concrete syntheses different than those used for experiment I. This way, it was possible to assess the extent to which the corresponding multifactorial model can be used for syntheses that lie outside the range of those been used for its estimation. In experiment II, 13 specimens were prepared for each of the concrete compositions. Water to cement ratio values were selected to be separated by equivalent intervals of 0.02 . At the same time superplasticizer's mix proportions were kept the same except from the mixture with the highest water to cement ratio. In this mixture a small decrease of superplasticizer's mix proportion was needed so the mixture did not become segregated. The cement content expressed in $\mathrm{kg} / \mathrm{m}^{3}$ was selected to be kept constant for the entire experiment, and as mentioned before, at a value different than the range used in experiment I. So, inevitably, it was impossible to change the water-to-cement ratio and keep the content of aggregates unchanged as expressed in $\mathrm{kg} / \mathrm{m}^{3}$. Otherwise the base for the calculation of constituents' content would not be in all cases equal to $1 \mathrm{~m}^{3}$. 
Additionally, ten specimen of composition I-G were prepared, half of which were tested at 7 days of age, while the rest were tested at the age of 14 days. These additional results where needed in order to apply the experiment II multifactorial model on the results of experiment I tests.

\begin{tabular}{cccccccc}
\hline Composition & $\begin{array}{c}\text { Cement } \\
{\left[\mathrm{kg} / \mathrm{m}^{3}\right]}\end{array}$ & $\begin{array}{c}\text { W/C } \\
{[-]}\end{array}$ & $\begin{array}{c}\text { Sand } \\
{\left[\mathrm{kg} / \mathrm{m}^{3}\right]}\end{array}$ & $\begin{array}{c}\text { Coarse aggregates } \\
4-16 \mathrm{~mm} \\
{\left[\mathrm{~kg} / \mathrm{m}^{3}\right]} \\
\text { Experiment I }\end{array}$ & $\begin{array}{c}\text { Coarse aggregates } \\
16-31.5 \mathrm{~mm} \\
{\left[\mathrm{~kg} / \mathrm{m}^{3}\right]}\end{array}$ & $\begin{array}{c}\text { Superplasticizer } \\
{\left[\mathrm{kg} / \mathrm{m}^{3}\right]}\end{array}$ & $\begin{array}{c}\text { Slump } \\
{[\mathrm{mm}]}\end{array}$ \\
I-A & 300 & 0.45 & 1021.2 & 623.1 & 341.3 & 4.20 & 10 \\
I-B & 300 & 0.50 & 1001.4 & 610.0 & 334.7 & 3.60 & 200 \\
I-C & 300 & 0.55 & 980.9 & 598.5 & 327.8 & 3.60 & 250 \\
I-D & 330 & 0.45 & 988.4 & 603.1 & 330.4 & 4.62 & 170 \\
I-E & 330 & 0.50 & 966.7 & 589.8 & 323.1 & 3.96 & 240 \\
I-F & 330 & 0.55 & 944.1 & 576.1 & 315.6 & 3.96 & 250 \\
I-G & 360 & 0.45 & 956.7 & 583.7 & 319.7 & 4.32 & 230 \\
I-H & 360 & 0.50 & 932.0 & 568.7 & 311.5 & 4.32 & 250 \\
I-I & 360 & 0.55 & 908.3 & 554.2 & 303.6 & 3.60 & 260 \\
I-J & 360 & 0.45 & 956.7 & 583.7 & 319.7 & 4.32 & 220 \\
& & & & Experiment II & & & \\
II-A & 280 & 0.46 & 1112.9 & 372.0 & 679.0 & 6.16 & 30 \\
II-B & 280 & 0.48 & 1104.7 & 369.2 & 674.0 & 6.16 & 70 \\
II-C & 280 & 0.50 & 1090.7 & 364.5 & 665.5 & 6.16 & 100 \\
II-D & 280 & 0.52 & 1088.2 & 363.7 & 664.0 & 6.16 & 140 \\
II-E & 280 & 0.54 & 1081.3 & 361.4 & 659.7 & 5.32 & 180 \\
\hline
\end{tabular}

Table 1: Mix compositions.

Slump test for each composition was performed according to EN 12350-2 and Slump test results are shown in Table 1. Curing procedure according to EN 12390-2 was followed. After demolding, specimens were immersed in water. The curing temperature was about $20^{\circ} \mathrm{C}$.

A number of different metal moulds, labeled with a serial number, were used to prepare the specimens for experiment I. These moulds were used in a randomized manner in order to prepare the specimens for all the various compositions presented in Table 1. The use of different moulds has led to an expected and reasonable dispersion of the geometric characteristics of the specimens (e.g. lack of surface flatness and perpendicularity, edge dimensional accuracy). Therefore, the following results are expected to be representative of reasonable dispersion of the geometric characteristics of the specimens been tested by a laboratory that uses any of these moulds and the parameter of the specimen's geometry is expected to have contributed into the results of this study.

Also, the uniaxial compression apparatus used was successfully calibrated with a subsequent correction of its calibration, according to International Standard ISO 7500-1. It can be assumed that the calibration of the apparatus contributes to the final combined uncertainty only when the corresponding systematic errors occur.

\section{METHOD OF ANALYSIS}

$\mathrm{E}$ xperiment II results were used into a multifactorial regression analysis procedure leading to a sigmoidal - by time equation:

$$
C S(W / C, t)=C S_{r e f} \cdot e^{\left[-(\tau / t)^{n}\right]}=\left(c_{0}+c_{1} \cdot(W / C)\right) \cdot e^{\left[-(\tau / t)^{n}\right]}
$$


The left part in the above Eq.(1), as described in Eq.(2).

$$
C S_{\text {ref }}=c_{0}+c_{1} \cdot(W / C)
$$

provides the value of compressive strength estimated for a reference curing age. In the case of Eq.(1) where the multifactorial model function incorporates the variation of $C S(W / C, t)$ for the entire range of curing age span, $C S_{\text {ref }}$ represents the tested specimens' compressive strength at infinite curing age, which could be called the final compressive strength $C S_{i n f}$. In the case of experiment I, Eq.(2) was used for testing specimens at a nominal curing age of about 28 days. The exponential part of Eq.(1) provides an estimation of the proportion of the final compressive strength reached at curing age t:

$$
P(t)=e^{\left[-(\tau / t)^{n}\right]}
$$

Sensitivity analysis and application of the law of propagation of uncertainty is easily performed according to the ISO GUM procedure when such a multifactorial function is used. Specifically, the sensitivity coefficients $C_{W / C}$ and $C_{t}$ can be estimated as the corresponding derivatives of the function in Eq.(1). These coefficients provide an assessment for the uncertainty of the result of concrete specimen compressive strength measurement which is attributed to the uncertainty in estimating the values for water-to-cement ratio and curing age, respectively. These two sensitivity coefficients are provided by the following equations:

$$
\begin{aligned}
& C_{W / C}=c_{1} \cdot e^{\left[-(\tau / t)^{n}\right]}=c_{1} \cdot P(t) \\
& C_{t}=\frac{C S(t) \cdot n \cdot \tau^{n}}{t^{n+1}}
\end{aligned}
$$

A laboratory performing testing in well-known concrete syntheses could use Eq.(1) as a baseline in the frame of quality control. That is, for concrete specimens that are similar in synthesis as those used for establishing Eq.(1), the result of any future compressive strength testing should not deviated significantly from the reference value provided by Eq.(1). For significantly different syntheses a laboratory should repeat the herein presented experimental procedure in order to fit Eq.(1) to the results of the corresponding compressive strength tests.

\title{
RESULTS AND DISCUSSION
}

\begin{abstract}
A ccording to experiment I results, the values $c_{0}=124 \pm 11 \mathrm{MPa}$ and $c_{1}=-150 \pm 22 \mathrm{MPa}$ were obtained for the least squares regression line of Fig.2a, with a satisfying fitting quality $\left(R^{2}=0.68\right)$. By interpreting the value obtained for parameter $c_{1}$, each percentage change in the water to cement ratio (i.e., a change in $W / C$ by 0.01 ), causes a change for the mean value of the compressive strength of the specimens by approximately $1.5 \mathrm{MPa}$. At the same time, no statistically significant correlation of $C S$ and $C C$ was detected based on experiment I results (Fig.2b). Fig.2a depicts the confidence bands of the regression line at the level of $95 \%$. But these limits are not expected to provide a generic conclusion as they correspond only to an experiment based on a similar set of specimens. In contrast, the prediction bands of the regression line can yield the $95 \%$ probability limits within which any single iteration of the test is expected to occur.

The regression procedure for experiment II compressive strength tests provided a statistically significant multifactorial function [see Fig.3a for the relation of the multifactorial model as a function of $W / C$ and Fig.3b for the relation of the model as a function of curing age $\theta$ with parameter values: $c_{0}=143 \pm 24 \mathrm{MPa}, c_{1}=-136 \pm 38 \mathrm{MPa}, \tau=0.45 \pm 0.18$ days and $n=$ $1.0 \pm 0.2$, at a confidence level of $95 \%$. The fitting quality is very satisfying $\left(R^{2}=0.92\right)$. One of the laboratory test results was omitted as an outlier, so 64 results were used in the regression procedure, instead of 65.

It should be noted that values for $c_{0}, c_{1}$ obtained from experiment II provide a linear function of $C S(t)$ with $W / C$, which provides an estimation of $C S_{i n f}$, i.e. $C s(t)$ for infinite curing age, when values for parameters $c_{0}, c_{1}$ obtained from experiment I provide a corresponding estimate, strictly dedicated to the nominal value of 28 days for curing age $t$. If a comparison between these two results is aimed at, then the right part of Eq.(1), the $P(t)$, should be taken into account.

The correlation of the compressive strength with the curing age parameter was strongly confirmed by the results of experiment II (Fig.3b). For this reason, Eq.(1) was applied to the results of experiment I tests, keeping the left part of the equation unchanged and, so limiting the regression process for optimizing the values for the sigmoidal shape parameters
\end{abstract}




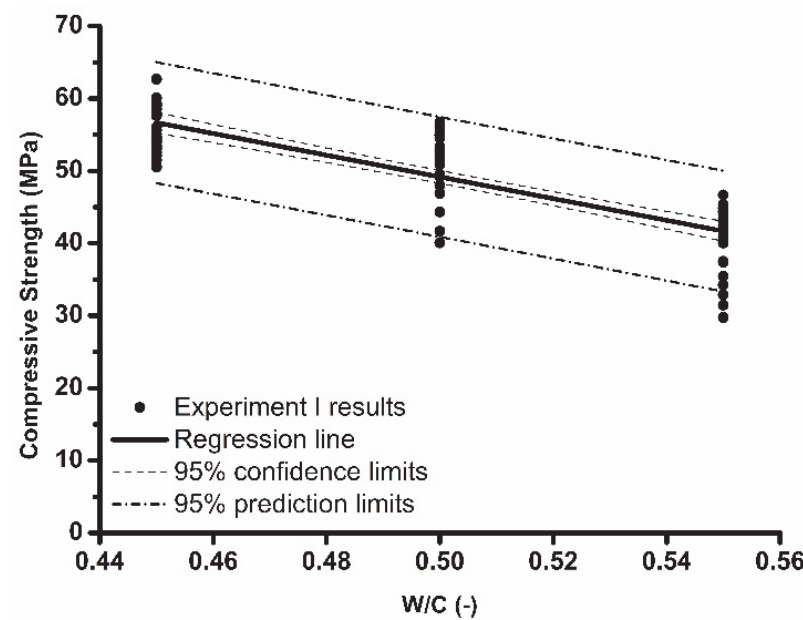

(a)

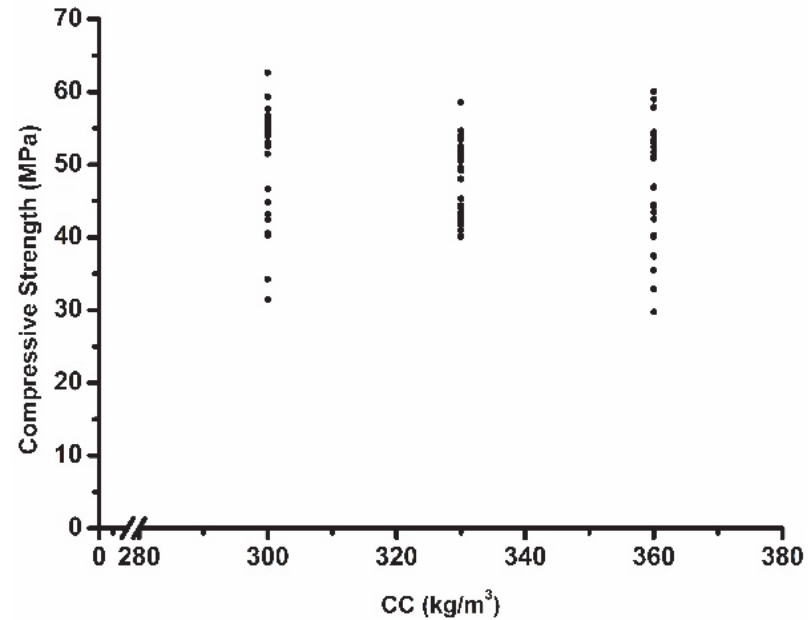

(b)

Figure 2: Evaluation of the effect of parameters (a) of water to cement ratio, $W / C$, and (b) cement content, $C C$, in determining the levels of compressive strength of specimens.

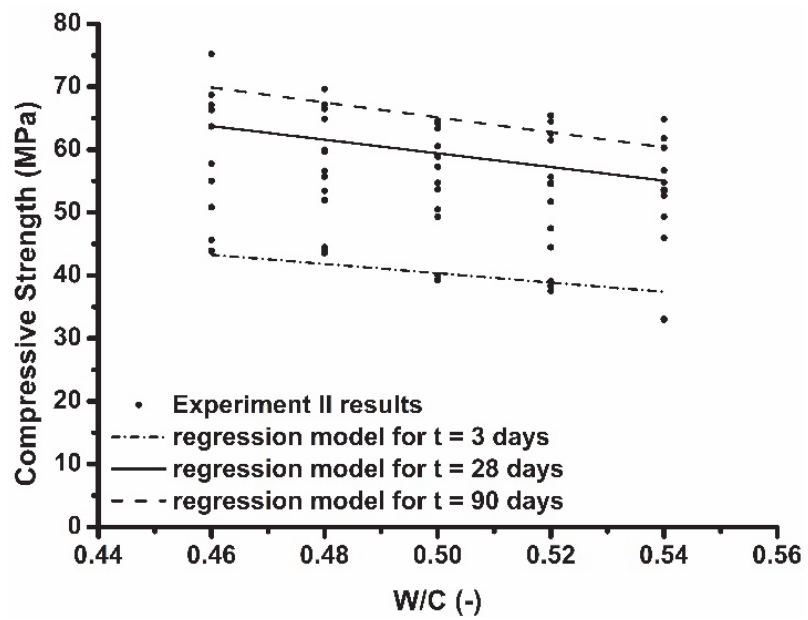

(a)

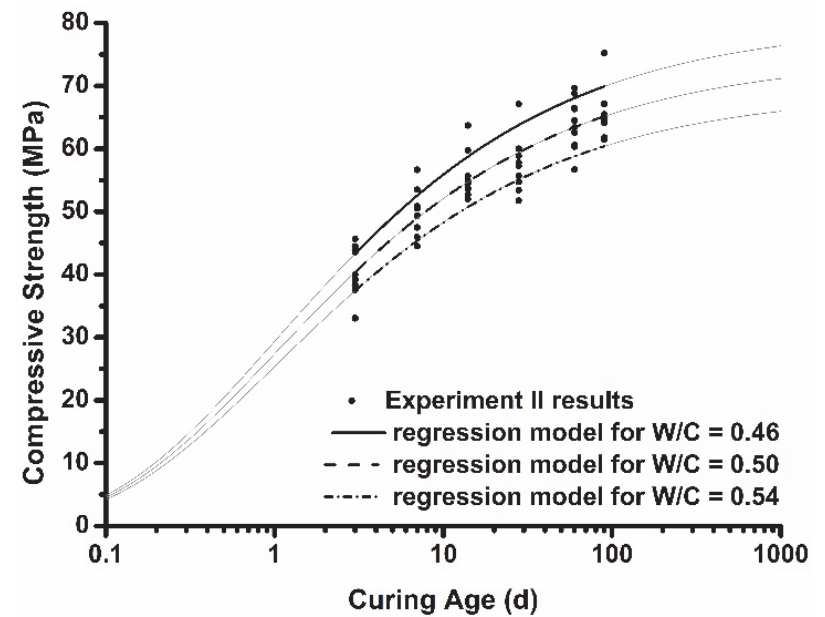

(b)

Figure 3: Multifactorial regression model as a function of (a) Water-to-Cement ratio $(W / C$ ) and (b) curing age $t$.

$\tau=3.8 \pm 0.9$ days and $n=0.50 \pm 0.08$, with a similarly very satisfying fitting quality $\left(\mathrm{R}^{2}=0.92\right.$, Fig.4a). It is of great importance that statistically significant parameter values where obtained, although the curing procedure was much slower than the one when experiment II was performed.

However, the correlation of the compressive strength with the curing age parameter, when the figure is narrowed to a curing age range of 27 to 31 days (Fig.4b for the results from experiment I), is not significantly apparent. It could be assumed that for such short time deviations, the correlation of compressive strength with curing age is a minor uncertainty factor for ages around the 28-day value. In contrast, as shown in Fig.2a, the significance of water to cement ratio, $W / C$, is confirmed for a range of values varying from 0.45 to 0.55 .

\section{Sensitivity analysis}

Despite the fact that experiment II regression function corresponds to specific qualitative characteristics of the constituents, the sensitivity analysis of this study is expected to have a more global validity. For example, using Eq.(4) on the results of experiment II, $C_{W / C}$ for curing ages of 7,28 and 90 days was estimated at $-89,-109,-119 \mathrm{MPa}$ per unit of $W / C$. This means that if we assume a maximum error on $W / C$ of about \pm 0.02 and a triangular distribution for a type $\mathrm{B}$ estimation of $\mathrm{W} / \mathrm{C}$ standard uncertainty, this would correspond to an effect on the compressive strength of the specimen of $0.7,0.9$ and 1.0 MPa, respectively. The same estimation based on Eq.(4) when performed for the results of experiment I provide the values of $-65,-94,-111 \mathrm{MPa}$ per unit of $W / C$ respectively, which correspond to standard uncertainties of $0.5,0.8$, and $0.9 \mathrm{MPa}$ for the final result on compressive strength measurement. 


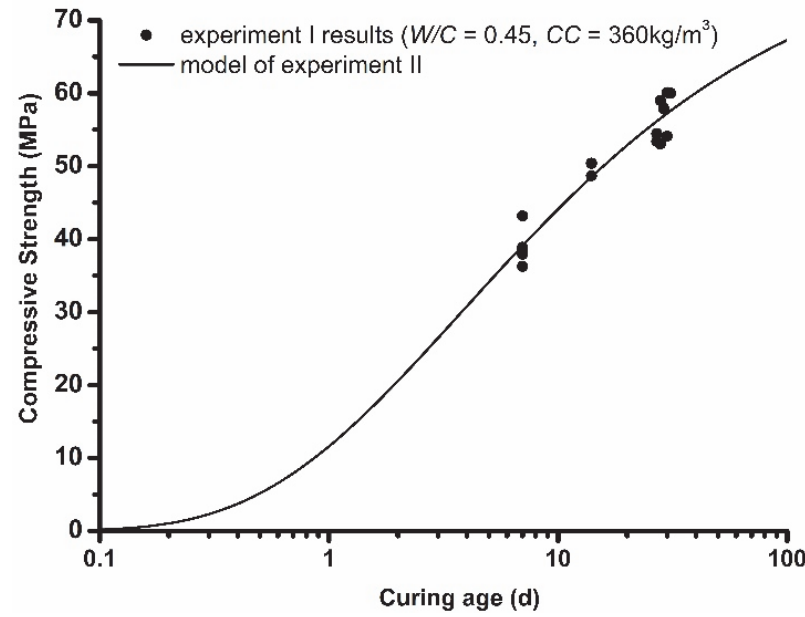

(a)

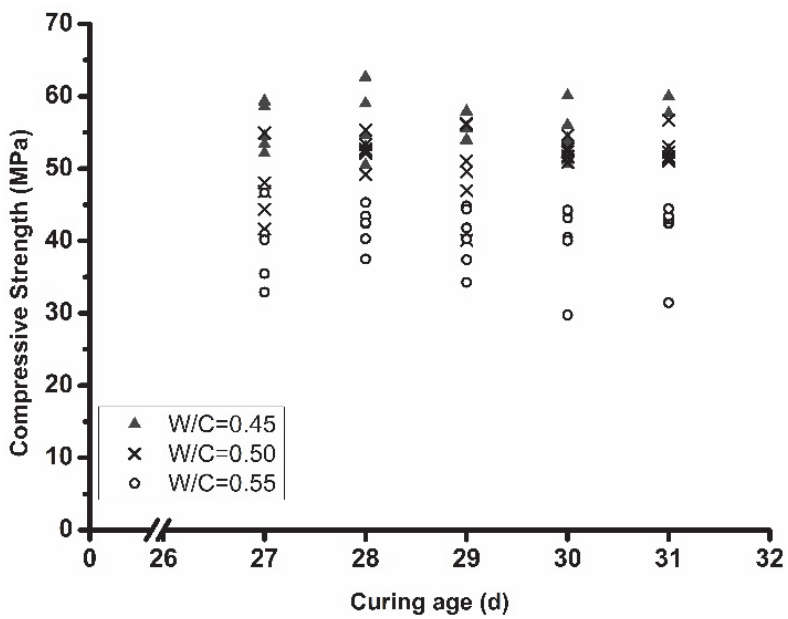

(b)

Figure 4: Effect of the specimen curing age on compressive strength test results: (a) for samples with $W / C=0.45$ and $C C=360 \mathrm{~kg} / \mathrm{m}^{3}$ and for ages from 7 to 31 days (the line shows the partial application of Eq.(1) as resulted from experiment II on these results from experiment I), (b) for samples of three different compositions and for curing ages varying from 27 to 31 days.

On the other hand, if we assume a maximum error on the curing age $t$ of about \pm 1 day and a triangular distribution for the corresponding type B estimation of standard uncertainty for variable t, Eq.(5) estimates an effect on the compressive strength of the specimen at $0.6,0.1$ and much less than $0.1 \mathrm{MPa}$ when experiment II results apply. The same estimation based on Eq.(4) when performed for the results of experiment I provide the values of 39, 57, 67 MPa per day, respectively, which correspond to standard uncertainties of $0.8,0.3$ and much less than $0.1 \mathrm{MPa}$ for the final result on compressive strength measurement.

It is interesting to compare these estimations to the results of experiment $\mathrm{I}$ where the expanded uncertainty $(\mathrm{k}=2$ ) for testing one single specimen according to the EN 12390 series procedure was estimated at about $17 \%$ for similar concrete syntheses. It is obvious that water to cement ratio and curing age errors cannot build up the major part of the testing procedure uncertainty. Major uncertainty parameters should be other like the geometry of the specimen, which is not easily assessed in an experimental way, and the compressive apparatus repeatability.

Application of Eq.(3) on the results of experiment II provides a value of about $80 \%$ of the final compressive strength of each specimen been reached at a curing age of 28 days. When applying Eq.(3) on the results of experiment I, this proportion is calculated at $69 \%$. Curing procedure was much slower in experiment I, which emphasizes the need to investigate the function of compressive strength by time.

It is also evident from Fig.2b that the compressive strength still increases significantly after the curing age of 28 days. When testing the specimens only at that curing age, independent for how many are the replicate specimens being used, the figure of $P(t)$ cannot be accomplished. The result will be always assessed on the basis of an assumed proportion of the final compressive strength been reached at 28 days. A laboratory, or a producer, wishing to estimate the final compressive strength of a series of specimens should apply Eq.(1). An interesting idea coming from this would be not to test 5 or 6 specimens at exactly 28 days, but testing them in consequent time intervals (e.g. 5, 10, 15, 20, 25 and 30 days) and thus producing the sigmoidal curve of Eq.(1). This would even provide directly the result on $C S_{i n f}$, with no need for $P(t)$ assumption.

Such semi-empirical models, especially if completed with all the significant parameters, are expected to be useful, among others, for accredited testing laboratories in order to perform their internal quality control program. Specifically, testing results lying outside the prediction bands of Eq.(1) should be considered as outliers.

Finally, one more possibility provided for performing quality control of compressive strength testing is that there is no strict bound for completing the test procedure at the exact nominal 28-days curing age. That means, if a laboratory misses to perform the test at exactly 28 days of curing age, or if a verification testing is to be performed at a significant time interval after the nominal 28-days curing age, it is feasible to reduce the test result by using the Eq.(1) to the corresponding value, at curing age equal to the nominal 28 -days.

\section{Prediction intervals}

When the regression line has been derived from pairs of values $\left[(W / C)_{i}, C S_{i}\right]$ of $\mathrm{i}=1, \ldots \mathrm{n}$ measurement results, then the prediction interval for the mean estimated $C S$ value out of Eq.(1), for a specific value of $W / C$, is calculated according to Eq.(6) [12]. 


$$
\widehat{C S} \pm t_{95 \%, n-2} \cdot \operatorname{se}(\widehat{C S})=\widehat{C S} \pm U_{\text {type } A}
$$

where

$$
s e(\widehat{C S})=s \cdot \sqrt{1+\frac{1}{n}+\frac{[(W / C)-\overline{(W / C)}]^{2}}{\sum_{1}^{n}\left[(W / C)_{i}-\overline{(W / C)}\right]^{2}}}
$$

while $\overline{W / C}$ is the average of all $(W / C)_{i}$ values from all measurements and $\widehat{C S}_{i}$ is the estimate for CS obtained for the specific $(W / C)_{i}$ value, using equation (1), and $s$ is given in Eq.(8):

$$
s=\sum_{1}^{n}\left(C S_{i}-\widehat{C S}_{i}\right)^{2}
$$

Based on experiment I, it would appear that the result of a single EN 12390-3 testing would lie within $\pm 17 \%$ of the mean value of compressive strength with a 95\% probability. This result should be broadly considered inherent for the process, the equipment and the operators that contributed, not taking into account the uncertainty posed by the compressive apparatus calibration procedure. Any reduction effort for these uncertainty parameters that are inherent for any laboratory performing the EN 12390-3 testing method requires optimization in one of these features of the test method.

\section{Opportunity for standardizing the procedure}

In a laboratory which performs compressive strength tests for concrete samples of a particular type of cement and aggregates, maintained at a specific temperature and tested at a certain curing age, a type A estimation of uncertainty for the test result can be made. In order to do that, a simple experiment, as follows, is needed to be designed and implemented:

(a) Multiple specimens are prepared for compositions that correspond to selected values of the $W / C$ and $C C$ parameters covering the range that the laboratory test method is performed for (e.g. such as the nine compositions presented in Table 1 for experiment I).

(b) The number of multiple specimens per composition will be derived by obtaining the product of the number of curing age values (when to perform the test) by the preferred number of specimen replicates per composition and curing age. For example, if five curing age values and two specimen replicates per each curing age value are selected, then ten samples per concrete composition are required. Curing ages for performing the corresponding compression tests should be selected for reasonable intervals by which the date of the test could deviate from the desired nominal value (i.e. the 28 days in the present study).

(c) To prepare the above test specimens, it is desirable to use as many different moulds as possible, for the purposes discussed in the preceding paragraph.

(d) By the above combination of samples, the coverage of all uncertainty parameters shown in Fig.1 is achieved, except for the possible systematic errors of the uniaxial compression device and the variation/deviation of the cement and aggregate characteristics.

(e) A statistical analysis of the test results for the compressive strength of the specimens, can be used to check whether such short deviations in the determination of the curing age and the cement content can influence the result.

(f) Applying the regression curve from Eq.(1) and Eqs.(2-4) for all the experimental results yields the uncertainty of the compression strength tests corresponding to the range of concrete compositions as per values selected in step a).

(g) According to ISO Guide for the expression of Uncertainty in Measurements [13], if the procedure is repeated under the same conditions and the result is obtained as the mean value of $n$ samples, the type A estimated standard uncertainty may be divided by $\sqrt{n}_{n}$. When conducting this uncertainty estimation, the standard uncertainty $U_{r s}$ attributed to load cell reference indications (i.e. the load cell used as a Reference Standard, RS, for the calibration of the test apparatus) shall be added, according to the law for error propagation. In Eq.(9), $U_{r s}$ is added after been divided by 2 , as an example of how the procedure should be applied when $U_{r s}$ is referred with a coverage factor $k=2$. Thus, an expanded uncertainty interval with a coverage factor $k=2$ is given in Eq.(9):

$$
2 \cdot \sqrt{\left(\frac{U_{t y p e} A}{2 \cdot \sqrt{n}}\right)^{2}+\left(\frac{U_{r s}}{2}\right)^{2}}
$$

According to Eq.(9), a laboratory that has performed an experiment like experiment I proposed in this study, estimating $U_{\text {ppeA } A}$, for testing according to EN 12390-3 on a single concrete specimen, at $17 \%$, using a calibration procedure for the test 
apparatus that is characterized by a reference standard uncertainty of $U_{r s}$ equal to $5 \%$, and also performing the EN 12390 3 testing method for $\mathrm{n}$ replicates of the sampled concrete, the corresponding expanded uncertainty is shown in Table 2.

The above estimations indicate that performing the EN 12390-3 method for more than $n=3$ replicates of the sampled concrete provide a minor optimization on the uncertainty of the results that comes as the mean value of the $n$ test results.

\begin{tabular}{cc}
\hline $\mathrm{n}$ & $\mathrm{U}_{\mathrm{CS}}[\%]$ \\
1 & 18 \\
3 & 11 \\
4 & 10 \\
6 & 9 \\
10 & 7 \\
\hline
\end{tabular}

Table 2: Indicative expanded uncertainty estimations.

\section{Quality control}

Testing compressive strength as per EN 12390-3 method, according to EN 12390-1 specimen definition, involves a great number of significant uncertainty parameters. Building an experiment that would be able to demonstrate accurately the effect of each parameter to the shape of the "vertical" distribution of the measurement results (e.g. see Figs.2,3) would demand so many combinations that would lead to a requirement for hundreds of specimens. Lack of homogeneity of specimens is one parameter that could cause strong effects to the shape of measurement results distribution, even when a statistical model is proven to fit them well. For instance, in Fig.2b, the greater dispersion in results shown for CCs equal to 300 \& $360 \mathrm{~kg} / \mathrm{m}^{3}$ could be attributed to an optimized (for such mixes) workability of mixes with CC equal to $330 \mathrm{~kg} / \mathrm{m}^{3}$. Skewness and kurtosis of the model residuals distribution as compared to the Gaussian could be a criterion for assessing whether the experiment results have been totally derailed.

Exclusion of outlying values should be addressed only in extreme cases as a negative skewness of the measurement results distribution, i.e. there are more extreme cases to the lower values as compared to the mean rather than to the higher values, could be normally explained. The reason for that could be the fact that for a given mix composition, there not a mechanism to produce an extremely high compressive strength performance for a corresponding specimen. On the other hand, lack of homogeneity occurs due to purely random reasons (e.g. a small spot inside the specimen's matrix where the constituents failed to be mixed properly) leading the specimen to fail during the compressive strength at relatively low compressive load.

In any case, a test for outliers could be also performed, i.e., based on the $99 \%$ prediction intervals of the CS to W/C linear correlation. This is achieved by substituting the $t_{95 \%, n-2}$ statistic in Eq.(6) by $t_{99 \%, n-2}$. This decision rule was applied at this study leading to the exclusion of three measurement results of experiment I that where lying outside the $99 \%$ prediction intervals for the linear correlation presented in Fig.2a.

Outlier analysis should be stricter in the case of the multifactorial model of experiment II, as this is its essential use. Depart from using Eq.(1) in order to perform a sensitivity analysis on the effect of C/W and curing age, it could be also used as a baseline for identifying outlying results when compressive strength tests are performed routinely on specimens coming from concrete compositions with the same characteristics as those used to build that baseline. The decision rule for the identification of outliers could be the expanded uncertainty, with a coverage factor equal to 3 , for the results provided when Eq.(1) is used.

\section{CONCLUSIONS}

he findings of the present study can be shortly summarized as follows: The mean compressive strength produced as a result of testing according to EN 12390-3 can be estimated by a sigmoidal-by-time multifactorial regression function incorporating both the water to cement ratio and curing age parameters. The use of this multifactorial function provides the opportunity to assess whether the compressive strength of the tested synthesis has a significant trend to increase after the nominal curing age of 28 -days.

The sensitivity coefficient of compressive strength as related to water to cement ratio is a function of curing age, specifically the relation of compressive strength with water to cement ratio is well represented by a line for which the slope 
changes in a sigmoidal relation with curing age. As related to curing age, this coefficient is also changing by time. At a curing age of 28 days it is expected to be non-significant. So, deviations in the order of a few days from the definition of 28 days do not affect significantly the compressive strength test result. The sensitivity on the effect of water to cement ratio and curing age uncertainty is minor as compared to the combined uncertainty of the test result at 28 days.

The results of this study are useful for a laboratory seeking accreditation on the method of EN 12390 series. If the laboratory decides to follow a type A estimation for the most of the uncertainty parameters, it is only needed to reproduce experiment I as a standardized procedure, using specimens that comply with the characteristics of concrete compositions that this specific laboratory is called to test according to EN 12390-3. Then this laboratory would gain an estimation of precision under partial reproducibility conditions. Reproducing experiment II would provide the laboratory, an additional opportunity to further evaluate the contribution of $\mathrm{W} / \mathrm{C}$ and curing age parameters on a sensitivity analysis basis.

As the laboratory repeats tests according to EN 12390 using the same apparatus, the same operators, for specimens with similar characteristics and for compositions falling within the range covered by the experiment, then the result of this type An uncertainty estimation can be used repeatedly. In the estimation resulting from $n$ such identical samples, the uncertainty parameter due to the systematic errors of the calibration of the test set should always be added.

It is estimated that in any of its applications, this method for a type A uncertainty estimating for a test specimen being tested according to EN 12390 will yield levels of uncertainty significantly greater than the expected repeatability of the device used, such as, for example, of $17 \%$ calculated in this paper.

The study could be further extended by proper experiments on a multifactorial sigmoidal curve incorporating also other significant parameters such as the curing temperature, the aggregates characteristics and the type of cement.

\section{NOMENCLATURE}

$C S(W / C, t) \quad$ concrete specimen Compressive Strength as a function of curing age $t$ and $W / C[\mathrm{MPa}]$

$C S_{r e f}$

$C S_{\text {inf }}$

$W / C$

$C C$

$t$

$P(t)$

\section{REFERENCES}

[1] Papadakos, G.N., Karangelos, D.J., Rouni, P.K., Petropoulos, N.P., Anagnostakis, M.I., Hinis, E.P., Simopoulos, S.E. (1892). Uncertainty in soil radioactivity measurement due to sampling definitional errors, Metrologia 2016: 6 $6^{\text {th }}$ National Biannual Conference in Metrology, Athens, Greece [in Greek].

[2] Féret, R.. On the compactness of the mortars, Annales des Ponts et Chaussées, 7(4), pp. 5-164.

[3] Abrams, D.A. (1927). Water-cement ratio as a basis of concrete quality, Journal of American Concrete Institute, 23, pp. 452-457.

[4] Freiesleben Hansen, P., Pedersen, J. (1985). Curing of Concrete Structures, CEB Information Bulletin, 166, 42.

[5] Carino, N.J. and Lew, H.S. (1983). Temperature effects on strength-maturity relations of mortar, Journal of the American Concrete Institute, Proceedings, 80(3), pp. 177-182.

[6] Yeh, C. (2006). Generalization of strength versus water-cementitious ratio relationship to age, Cement and Concrete Research, 36, pp. 1865-1873. DOI: 10.1016/j.cemconres.2006.05.013

[7] Metwally abd allah, A. (2014). Compressive strength prediction of Portland cement concrete with age using a new model, HBRC Journal, 10(2), pp.145-155. DOI: 10.1016/j.hbrcj.2013.09.005

[8] Gavela, S., Nikoloutsopoulos, N., Papadakos, G., Passa, D., Sotiropoulou, A. (2018). Multifactorial experimental analysis of concrete compressive strength as a function of time and water-to-cement ratio, $1^{\text {st }}$ International Conference of the Greek Society of Experimental Mechanics of Materials, Structural Integrity Procedia 10 (2018) 135-140. 
[9] Gavela, S., Papadakos G., Kaselouri-Rigopoulou V. (2017). A suggestion for standardizing a traceable process for the determination of the mechanical properties of concrete containing thermoplastic polymers as aggregates, In: Thermoplastic Composites-Emerging Technology, Uses and Prospects, Materials Science and Technologies NOVA publications book, ISBN: 978-1-53610-727-2

[10] Sotiropoulou, A., Gavela, S., Nikoloutsopoulos, N., Passa, D., Papadakos, G. (2017). Experimental study of wood shaving addition in mortar and statistical modeling on selected effects, Journal of the Mechanical Behavior of Materials, 26, pp. 1-2. DOI: 10.1515/jmbm-2017-0013

[11] EURACHEM/CITAC Guide (QAC 2016). Guide to Quality in Analytical Chemistry - An Aid to Accreditation, 3rd edition.

[12] Gavela, S., Papadakos. G., Nikoloutsopoulos, N., Passa, D., Sotiropoulou, A. (2018). Determination of type A uncertainty for the concrete compressive strength test, $18^{\text {th }}$ Panellenic Concrete Conference, Athens [in Greek].

[13] Simopoulos, S.E. (2003). Technical Measurements, Handouts for the corresponding lesson - NTUA School of Mechanical Engineering, Athens. Available at: http://nuclear.ntua.gr.

[14] BIPM, Evaluation of measurement data - Guide to the expression of uncertainty in measurement, JCGM 100: 2008 (GUM 1995 with minor corrections) 